\title{
Distance transactionnelle et centres de ressources en langues
}

\author{
Eglantine Guély
}

ATILF équipe CRAPEL.

Université Nancy 2

3 place Godefroi de Bouillon

54015 NANCY CEDEX

Eglantine.Guely@univ-nancy2.fr

\section{RÉSUMÉ}

Cet article vise à établir des rapports entre une théorie issue de la FAD, la distance transactionnelle et ses variables de structure, dialogue et autonomie, avec la conception d'un type de dispositif propre à la formation en langue : le centre de ressources (CRL). Pour cela, nous tenterons d'appréhender ces différentes notions et de les mettre en rapport avec un type d'accompagnement pédagogique pratiqué dans les CRL, le conseil à l'apprentissage. Nous étudierons le cas d'un CRL particulier dont nous tenterons d'évaluer le degré d'ouverture et de décrire l'état prescrit. Cette phase devrait nous permettre de circonscrire une zone délimitant les possibilités de choix offertes aux apprenants dans ce dispositif, c'està-dire une zone d'actualisation potentielle.

\begin{abstract}
This article aims at establishing relationships between transactional distance theory, which articulates the variables of structure, dialog and learner autonomy, and the conception of a specific language learning system: a self-access centre. We will try to establish a theoretical frame for these notions, and use it to describe a professional gesture typical of self-access centres : language advising. We will study the case of a peculiar self-access centre appropriation and openness. We expect that this elaboration of the system will help us to limit a zone of learners' possibilities of choices: a potential actualisation zone.
\end{abstract}

MOTS-CLÉS : autonomie, auto-direction, contrôle, structure, ouverture, dialogue, conseil, centre de ressources en langues, distance transactionnelle, dispositif

KEYWORDS: autonomy, self-direction, control, structure, openness, dialog, advising, selflearning system, self-access centre, transactional distance, language learning system. 


\section{Introduction}

L'intérêt de considérer la distance en formation peut résider dans l'intention de l' " apprivoiser » (Jacquinot, 1993, 56), c'est-à-dire d'optimiser la conception d'un dispositif de formation pour que ses composantes techniques, matérielles et humaines le réduisent au minimum d'effets négatifs, ou de l'« assumer » (Bernard, 1999, 35), c'est-à-dire de profiter de la conscience des écarts, des séparations, non seulement pour optimiser la conception du dispositif, mais aussi pour prendre en compte, à tous les niveaux, la dimension de la personne en formation et les processus de mise à distance ou d'appropriation qui peuvent la concerner au cours de son apprentissage.

En tentant d'abord de rapprocher la notion d'ouverture de la structure de Centres de Ressources en Langues ( $C R L)$, et en tentant de décrire les spécificités des interactions pédagogiques au sein de ce type de dispositifs, nous chercherons à construire un cadre d'analyse susceptible d'étudier l'appropriation d'un CRL par les apprenants qui le fréquentent. Notre hypothèse de travail étant, qu'avec une structure ouverte, une visée d'autonomisation de l'apprenant et un accompagnement spécifique et modélisé, la distance transactionnelle inhérente à un $C R L$ devrait être de faible amplitude, et les possibilités d'appropriation (Rabardel, 1995 ; Paquelin, 2004, 2007) et de co-construction du dispositif par l'apprenant importantes. Le terrain d'analyse, un dispositif d'apprentissage de langue anglaise, nous permettra d'aborder un premier élément de description : le dispositif en tant qu'état " prescrit ". Nous essaierons d'appréhender la vision des concepteurs, pour construire des hypothèses et des perspectives de recherche sur l'actualisation possible de ce dispositif par ses acteurs, dans le cadre d'une démarche d'individualisation autonomisante des apprenants.

\section{Distances et formation : la distance pédagogique en question}

La distance est une notion à la fois "spatiale, temporelle, mais aussi technologique, psycho-sociale, et socio-économique » (Jacquinot, 1993, 57). En formation, celle qui semble la plus problématique est celle " qui sépare celui qui veut ou doit apprendre et celui qui sait et veut ou doit enseigner, c'est-à-dire la distance pédagogique » (Jacquinot, 1993, 60). Cette distance entre formé/formateur ou formé/prescripteur apparait comme une évidence à prendre en compte dans le contexte de la formation à distance ou dans la conception de supports pédagogiques destinés à des apprenants " non-accompagnés ». Pourtant la présence physique des apprenants et des formateurs ne garantit pas une "présence psychologique » (Jacquinot, 1993, 60) de ceux-ci, encore moins une proximité dans leurs relations, leurs échanges ou la vision de leurs projets. Une forme de distance transactionnelle, c'est-à-dire un " écart perceptuel/communicationnel » existe dans toute situation de formation, même en face-à-face (Moore, 1993). Cet écart perceptuel et communicationnel se mesurerait à "la présence (ou à l'absence) d'un dialogue éducatif d'une part, et à la présence (ou à l'absence) d'une structure plus (ou moins) contraignante, d'autre part » (Bouchard, 2000, 69), la relation entre ces variables étant que plus une formation est structurée, et moins elle n'offre de possibilité de dialogue entre formateur et apprenant, plus la distance transactionnelle entre apprenant et formateur est importante ${ }^{1}$.

\footnotetext{
1 "When a programme is highly structured and teacher/learner dialogue is non-existent the transaction between learners and teachers is high."
} 
Une distance transactionnelle importante conduirait enfin à une nécessité également importante d'exercice de l'autonomie pour l'apprenant ${ }^{2}$ (Moore, 1993, 27). Ces variables et leurs relations ont été largement étudiées, et des critiques sérieuses ont été émises quant à la validité de cette théorie de la formation à distance. Gorsky et Caspi (2005) relèvent par exemple les conclusions de différentes études, et pointent des incohérences théoriques, qui relèvent essentiellement du manque de définition opérationnelle des différents concepts. Leur analyse réduit les paramètres influents sur la distance transactionnelle au seul dialogue, hiérarchiquement dépendant de la variable structure et déterminé par les caractéristiques de l'apprenant, dont son autonomie. Ils réduisent ainsi la notion de distance transactionnelle à celle d'incompréhension, le dialogue étant interprété comme le facteur essentiel favorisant la compréhension ${ }^{3}$ (Gorsky et Caspi, 2005, 7-8). II ne reste pour autant que la compréhension d'un système de formation par l'apprenant reste une question complexe. L'appareillage théorique construit par Moore peut être exploité une fois des définitions opérationnelles des termes structure, dialogue, autonomie explicitées (Jézégou, 2007). Nous reviendrons ainsi sur celles qui fondent notre analyse pour établir un protocole visant à appréhender les phénomènes d'appropriation et d'actualisation en jeu au cours de la formation, et à figurer des écarts significatifs entre différents états de ces processus. Si la structure d'un système est souple, flexible, ouverte, elle peut peut-être permettre d'autant plus à l'apprenant d'en choisir des composantes, et par là même de « co-construire » avec les formateurs ses savoirs et son propre système particulier de formation (Paquelin, 2004, 160). C'est dans ce cadre que nous allons à présent tenter de rapprocher les notions de structure - et d'ouverture, à celles de dialogue et d'autonomie des pratiques des CRL pour construire des éléments d'analyse de l'appropriation d'un CRL particulier.

\section{Structure, ouverture et Centres de Ressources en Langues (CRL)}

La notion de structure renvoie chez Moore à la question de la rigidité ou de la flexibilité des objectifs, des stratégies d'enseignement et des méthodes d'évaluation d'un programme de formation. Cette notion sert à décrire l'étendue de la capacité d'un programme à s'accommoder ou à répondre aux besoins individuels de chaque apprenant (Moore, 1993, 26) : "Structure expresses the rigidity or flexibility of the programme's educational objectives, teaching strategies, and evaluation methods. It describes the extent to which an education programme can accommodate or be responsive to each learner's individual needs."

La notion de structure a été rendue opérationnelle en particulier par le travail de Jézégou (Jézégou, 2004, 2005; 2007,), qui explicite la question de la flexibilité d'un programme de formation selon trois grandes logiques de construction de l'environnement d'apprentissage : auto-structuration (totalement créé par l'apprenant en dehors de tout cadre institutionnel), hétéro-structuration (entièrement conçu et prédéterminé par l'institution), et co-structuration (co-créé et négocié par apprenant et institution selon les ressources disponibles et les contraintes). La flexibilité renvoie ainsi «principalement au degré de libertés de choix ouvert à l'apprenant dans la détermination des différentes composantes de son environnement

\footnotetext{
2 «the greater the structure and the lower the dialogue in a programme the more autonomy the learner will exercice »

3 " "structure" is a variable that determines to some degree the extent of "dialogue" which, in turn, determines the extent of "transactional distance" (...) "learner autonomy" is a variable that also determines to some degree the extent of "dialogue" which (...) determines the extent of "transactional distance". (...)

The theory may be construed as the tautology : "As understanding increases, misunderstanding decreases."
} 
éducatif » (Jézégou, 2005, 2006 in 2007, 346) et se rapproche de la notion d'ouverture : un dispositif offrant de larges libertés de choix permettant à l'apprenant de structurer lui-même son environnement d'apprentissage est hautement ouvert, alors qu'à l'inverse une forte hétéro structuration relève d'un environnement fermé (Jézégou, 2007, 346).

Les CRL sont des dispositifs d'apprentissage, " des espaces où se trouvent des ressources matérielles : salles de travail, équipement technologique, documents variés sur divers supports techniques », pourvu également de « ressources humaines : personnels d'accueil, conseillers techniques " voire dans certains cas "tuteurs et/ou formateurs " (Glikman, 2002, 200). Certains CRL peuvent être considérés comme des structures ouvertes, comme celui analysé par Poteaux $(2007,78)$ qui « propose une formation organisée autour de supports pédagogiques variés (dont les TICE), avec des modalités de travail diversifiées, seul, en groupe, avec ou sans enseignant, offrant une souplesse de fréquentation et d'horaires ». Les CRL peuvent en effet être conçus selon différents modèles et conceptions de l'apprentissage. La classification d'Albero (2000, in Glikman, 2002, 206) se fonde, comme l'explicitation des notions de flexibilité et d'ouverture chez Jézégou, sur le degré de contrôle accordé à l'apprenant sur sa formation, à travers quatre dominante observables : prescriptive, tutorale, coopérante et autodirective. Si l'on considère à présent la notion d'ouverture des dispositifs de formation, selon leur propriété: « d'ouvrir à l'apprenant des libertés de choix, afin qu'il puisse exercer un contrôle socio-organisationnel et pédagogique sur sa formation et sur ses apprentissages » (Jézégou, 2005, 103), il apparait que selon ces différents modèles, l'amplitude de l'ouverture des CRL pourra être variable. Cette variable de l'ouverture peut être évaluée : elle " se conçoit en définissant le degré de liberté de choix accordé à l'apprenant pour chacun des facteurs contextuels du dispositif : temps, lieu, accès, objectifs, rythme, supports pédagogiques multimédias, moyens de communication, personnes-ressources, évaluation, etc. " (Jézégou, 2002, 52). Le protocole développé par cette auteure (Jézégou, 2004, 2005, 2007) sera ainsi utilisé pour délimiter l'ouverture du dispositif étudié et tenter de quantifier le degré de flexibilité de sa structure.

\section{Autonomie, auto-direction, contrôle et centres de ressources en langues}

Selon Moore $(1973,666)$, la formation à distance s'intéresse nécessairement à des apprenants indépendants, et les met en relation avec l'institution éducative via un système de communication. Le terme indépendant comme le terme autonome, renvoient chez Moore à l'exercice d'un degré sensible de responsabilité, de contrôle de l'apprenant sur les décisions qui concernent son apprentissage. La formation à distance implique selon lui un relativement haut degré d'autonomie de l'apprenant par la posture solitaire de celui-ci au moment d'apprendre, qui l'oblige à accepter d'assumer un haut degré de responsabilité dans la conduite de son apprentissage, et ce quelque que soit le type de formation proposée ${ }^{4}$. Ce contrôle exercé par l'apprenant est à rapprocher de la notion de contrôle psychologique défini par Long (1989), qui considère que " l'appropriation de l'acte d'apprendre est indépendante de l'environnement dans lequel elle s'articule » (Bouchard, 2000, 69). Jézégou

\footnotetext{
4 "Because he is alone, perhaps in a non-individualised, ant therefore self-pacing, program (perhaps without dialogue, because he may be very distant from his teacher), the learner is compelled to accept a comparatively high degree of responsibility for the conduct of his learning program." (Moore, 1973, 666)
} 
explicite cet ancrage en rappelant que Long « a toujours accordé un poids prépondérant au contrôle psychologique au point d'affirmer qu'il constitue la condition nécessaire et suffisante de l'apprentissage autodirigé " et ajoute que le "contrôle pédagogique exercé par le dispositif sur les situations d'apprentissage " joue aussi un rôle important dans la mise en œuvre de l'autonomie de l'apprenant (Jézégou, 2008, 347). En effet, si la prise de contrôle sur la formation par l'apprenant est possible dans un environnement hétéro dirigé, parce que " la personne a une tendance innée à s'auto actualiser et à s'autoréguler dans ses processus de développement ", cela n'empêche que "cette capacité peut être favorisée, freinée ou totalement paralysée par l'environnement. Un contexte rigide, imposé, où la personne a peu de liberté de mouvement ou de choix, aura pour effet de ralentir le mouvement d'actualisation et de développer des attitudes de dépendance chez le sujet ». (Jézégou, 2005, 104). Cette perspective nous semble importante à prendre en compte pour tenter de transposer la notion de distance transactionnelle du contexte de la Formation $\mathrm{A}$ Distance (FAD) à celui des Centres de Ressources en Langues. En effet, si l'on définit le contrôle psychologique comme " un processus intérieur et spécifique à chacun " se référant à « deux dynamiques fondamentales : la motivation (...) et la cognition » (Jézégou, 2002), et le contrôle pédagogique uniquement sous l'angle des libertés de choix accordées à l'apprenant, la variable dialogue ne peut être prise en compte, la notion de distance transactionnelle ne s'avèrerait que partiellement exploitée et celle d'autonomie encore insuffisamment développée.

Si l'on redéfinit à présent la mise en œuvre de l'autonomie d'apprentissage en tant que contrôle pédagogique de la formation par l'apprenant, c'est-à-dire le fait d' «avoir la responsabilité, et l'assumer, de toutes les décisions concernant tous les aspects de cet apprentissage, c'est-à-dire : la détermination des objectifs, la définitions des contenus et des progressions, la sélection des méthodes et techniques à mettre en œuvre, le contrôle du déroulement de l'acquisition proprement dite (rythme, moment, lieu, etc.), l'évaluation de l'acquisition réalisée » (Holec, 1979, 32), les formations en auto-direction ne seraient accessibles qu'à des apprenants déjà formés à cette pratique pédagogique. Or, la perspective d'autonomiser les apprenants au fil de la formation est une intention forte des concepteurs et des formateurs de CRL (Holec et Cembalo, 1973, 38), et la structure de ce type de dispositif ainsi que les accompagnements pédagogiques proposés devraient pouvoir participer d'une mise en œuvre de l'autonomie de l'apprenant : « Ces systèmes mettent ainsi en place des interactions multiples, matérielles et humaines, qui permettent à l'apprenant de confronter son système de représentations à des situations nouvelles. Cela participe du développement de l'apprendre à apprendre », (Ciekanski, 2005, 54). Les rapports entre contrôle psychologique et contrôle pédagogique dans la capacité des individus à exercer leur autonomie en apprentissage peuvent être considérés grâce à l'éclairage de la notion d'ouverture, mais aussi à travers l'analyse des médiations en cours dans les formations, et des places et rôles des différents acteurs.

\section{La notion de dialogue pédagogique et le conseil à l'apprentissage}

La notion de dialogue renvoie dans la théorie de la distance transactionnelle à l'interaction entre l'enseignant et l'apprenant, quand l'un donne des consignes et que l'autre répond. D'autre part, le terme dialogue est utilisé pour souligner les qualités que ce type d'interaction doit avoir : un dialogue a un but, est constructif, valorisé par les acteurs, vise à favoriser la 
compréhension de l'apprenant. La relation entre les interlocuteurs est symétrique : chacun fait preuve d'une écoute respectueuse et active, chacun contribue à la co-construction du sens : "Each party in a dialogue is a respectful and active listener; each is a contributor, and builds on the contributions of the other party or parties " (Moore, 1993, 24). Moore a également observé que "le processus d'appropriation (de l'apprentissage) se développe chez l'apprenant de façon dialogique, c'est-à-dire que l'internalisation des buts et des objets de l'apprentissage se forme progressivement par le biais de questionnements, de remises en question et de reformulations qui nécessitent une médiation par le dialogue » (Bouchard, 2000,70 ). Autrement dit, le processus d'appropriation de la démarche d'apprentissage résulte d'une certaine distanciation cognitive, une capacité mentale d'abstraction et de réflexivité, nécessaire pour "prendre conscience des mécanismes de sa propre pensée » et les améliorer et les piloter, or cette métaconduite doit être apprise et entrainée systématiquement (Linard, 2003, Poteaux 2007). Ces caractéristiques qualitatives ne suffisent pourtant pas encore à rendre opérationnelle la notion de dialogue, c'est pourquoi Jézégou (2007, 348-349) s'appuie sur le modèle de la présence (Garrison et Anderson, 2003) pour tenter de construire des indicateurs plus précis de la qualité du dialogue, entre formateurs et apprenants, mais aussi entre apprenants. Ce modèle de la présence recouvre trois dimensions : sociale, cognitive et éducative. Le modèle de Moore, restreint au terme " dialogue pédagogique » s'en retrouve ainsi étoffé, et des critères plus précis d'analyse d'entretiens de conseil peuvent être construits pour rechercher des indicateurs de ces trois niveaux de présence. D'après Ciekanski (2007, 119-120) les entretiens de conseil présentent une structure régulière, contenant toujours trois types de séquences: des séquences pédagogiques, organisationnelles et conversationnelles, qu'il serait intéressant d'analyser selon ces niveaux de présence.

Nous suivrons à présent l'hypothèse que " la difficulté à passer des CRL à l'EAD ne réside (...) ni dans la configuration des ressources, ni dans leur accès, (...) mais davantage dans la mise en scène des dimensions sociales du processus d'apprentissage. C'est peutêtre vers les représentations de l'absence et de la présence dans les modèles pédagogiques à l'œuvre en lien avec la place et le rôle impartis aux acteurs du dispositif qu'il faut tourner le regard » (Poteaux, 2007, 87). Nous rejoignons en cela également l'hypothèse de Gorsky et Caspi $(2005,8)$ qui situent le dialogue dans une position de dépendance de la structure.

\section{Rôles et compétences des conseillers dans les CRL}

Revenus de l'illusion que « proposer des environnements d'apprentissage diversifiés » ne suffit pas « pour que les apprenants s'en emparent spontanément en y faisant leur choix et en y construisant leurs parcours » les personnels pédagogiques intervenant dans les centres de ressources sont arrivés à "une conception plus complexe des relations que les apprenants souhaitent ou sont capables d'établir à un moment ou à un autre entre le processus d'apprentissage et le processus d'enseignement » (Puren, 2004, 241), c'est-à-dire qu'ils situent leur action à différents plans possible, en fonction de l'apprenant et de la situation, sur le continuum : faire apprendre / enseigner à apprendre / enseigner à apprendre à apprendre / faciliter l'apprendre à apprendre / laisser apprendre (Puren, 2004, 244). Le transfert de contrôle pédagogique de l'enseignant vers l'apprenant est ainsi observable à travers ces actes, qui peuvent être aussi considérés comme des étapes dans la construction de la compétence d'apprentissage de l'apprenant, mais aussi comme des 
indicateurs d'une co-construction possible du micro-système d'apprentissage de l'apprenant. La négociation apprenant/formateur concernant le rôle de l'accompagnant est ainsi une caractéristique forte des entretiens de conseil à l'apprentissage ${ }^{5}$ (Ciekanski, 2007, 125).

Pour modéliser le champ d'intervention du conseiller, rappelons tout d'abord que ses tâches pédagogiques sont généralement de trois ordres : accompagnement individuel au cours d'entretiens de conseil, animation d'ateliers méthodologiques ouverts à des groupes d'apprenants, mise à disposition de ressources aux apprenants (recherche de matériels, intégration de nouvelles ressources au catalogue du centre, conception de supports pédagogiques), (Mozzon-McPherson, 2007, 77). Nous nous intéresserons plus particulièrement à l'interaction de conseil, où le conseiller agit à trois niveaux : ceux de l'apport conceptuel (culture langagière et culture d'apprentissage), de l'apport méthodologique (propositions de documents, d'activités, d'éléments du programme) et du soutien psychologique (Gremmo, 1995, 50 ; Carette et Castillo, 2004). C'est-à-dire que le conseiller est sensé permettre à l'apprenant de prendre le contrôle de sa formation sur le plan pédagogique, en l'aidant à opérer les choix relatifs au projet de formation (objectifs, contenus, procédures d'évaluation, rythmes, temps, lieux, etc.) et à franchir le pas entre le potentiel et le réel, entre le fait d' « avoir les capacités à prendre le contrôle de sa formation » et celui d'" exercer réellement ce contrôle en situation ». (Jézégou, 2005). Cette pratique requière un changement d'attitude radical de la part de l'enseignant/conseiller comme de l'apprenant et des compétences spécifiques pour gérer la complexité de la tâche : "For advising to be effective it requires a significant shift in attitude on the part of both the adviser and the learner, and a specific competence to deal with the complexity of the task. " (Mozzon-McPherson, 2007, 77).

Ces compétences sont d'une part d'ordre pédagogique, les conseillers ayant besoin de sérieuses connaissances théoriques et pratiques sur l'apprentissage et l'acquisition des langues étrangères ${ }^{6}$ (Mozzon-McPherson, 2007, 78). Le conseiller apporte une expertise pédagogique, sans pour autant décider (Gremmo, 1995) ni contrôler. Ce point essentiel correspond bien à la conception de l'autonomie de Moore, qui se réfère à Allen Tough en distinguant l'aide apportée, qui peut être considérable, du contrôle ${ }^{7}$ (Moore, 1973, 177-178). De plus, face à des apprenants généralement fortement ancrés dans une culture d'apprentissage hétéro dirigée, un déconditionnement est souvent nécessaire pour que ceux-ci acceptent de prendre le contrôle sur les modalités de leur apprentissage. En effet, ceux-ci ont besoin de " se libérer de l'idée qu'il existe une méthode idéale » et " que les enseignants détiennent cette méthode 》 (Holec, 1979, 117). De nombreuses représentations sur l'apprentissage ou les langues peuvent émerger au cours des entretiens, et le dialogue entrepris avec le conseiller peut concourir à faire évoluer ces représentations. Le conseiller doit ainsi parvenir non pas à expliciter ses propres connaissances sur l'apprentissage et l'acquisition, mais à susciter la parole de l'apprenant et à construire des

\footnotetext{
${ }^{5}$ « it appears that even if advisers share the same professional definition of what an advising relationship is, this definition is constantly renegociated in relation to the context and to each learner »

6 "advisers need to possess a sound theoretical and practical knowledge and understanding of language learning and second language acquisition"

7 "The helper provides detailed information about the various possibilities that are open, but lets the learner himself make the decisions. (...) The distinction between help and control is important, because it helps us realize that a learner can receive a great deal of help without giving up any of his control, or responsability."
} 
propositions à partir de celle-ci, et ce dans le langage utilisé par l'apprenant ${ }^{8}$ (MozzonMcPherson, 2007, 78), ce qui peut permettre de faire perdre au discours de sa "distance didactique au profit d'un souci de proximité cognitive de l'étudiant »(Poteaux, 2007, 82). Conseillers et apprenants peuvent ainsi, grâce au dialogue, co-construire un " terrain commun " et des "critères fonctionnels " utiles à l'apprenant pour sa prise de décision (Gremmo, 1995, 42). Le conseiller doit donc également posséder des outils de gestion du dialogue et de l'interaction, telle que l'écoute active, des techniques de pause, de gestion de l'intonation pour susciter des approfondissements ou des éclaircissements de la pensée de l'apprenant ${ }^{9}$ (Mozzon-McPherson, 2007, 77). Des qualités du dialogue pédagogique, à savoir le respect de relations symétriques dans les échanges et le soutien à la distanciation cognitive et à l'élaboration de nouveaux schèmes concernant les procédures et les conceptions de l'apprentissage, devraient ainsi pouvoir être observés dans des entretiens de conseil d'un CRL à dominante autodirective. Ces qualités apparaissent être fortement liées aux compétences des conseillers sur le plan pédagogique d'une part, mais aussi sur le plan interactionnel et relèvent des trois niveaux de présence : expertise et présence pédagogique, soutien à la distanciation et présence cognitive, gestion de l'interaction et présence sociale.

La conception de l'autonomie et de l'autodirection dans les centres de ressources est donc centrale, puisqu'elle conditionne la conception de la structure des dispositifs à vocation autonomisante ainsi que la modélisation de l'accompagnement proposé.

\section{Etude de cas : dispositif prescrit et ouverture}

La genèse de notre projet de recherche s'inscrit dans celle d'un nouveau dispositif d'apprentissage de l'anglais, qui résulte de la collaboration entre un mandataire du secteur privé avec l'équipe du $\mathrm{CRAPEL}^{10}$. Ce dispositif a été conçu pour répondre à l'objectif de commercialiser un service de formation à la langue anglaise destiné à $80 \%$ à des particuliers, adultes, et de concevoir ce service comme un centre de ressources pour l'apprentissage de la langue anglaise, pourvu d'une numérisation optimale des ressources et d'un accompagnement pédagogique adapté à une démarche d'individualisation autonomisante de l'apprentissage (Jézégou, 2005). Le centre offrira ainsi des ressources matérielles et humaines que l'apprenant pourra exploiter au cours de son apprentissage. Les ressources matérielles seront centralisées via un site Internet conçu pour permettre l'accès à une base de données des supports disponibles - documents authentiques vidéos, audios et textes, méthodes d'apprentissage sur CD-Rom ou en ligne, DVD. Selon l'orientation méthodologique choisie, c'est-à-dire une dominante autodirective, le site Internet aura pour fonction d'assister la démarche d'apprentissage de l'apprenant en étant pourvu de rubriques et de conseils favorisant la construction du parcours d'apprentissage de l'apprenant. Celui-ci pourra ainsi, à l'issue d'entretiens de conseil ou de manière autonome, définir ses besoins et ses objectifs de travail via un sous-système dédié, accéder automatiquement à des ressources liées à ses objectifs dans la base de données, réaliser des auto-évaluations,

\footnotetext{
8 "One of the qualities of a good adviser is not to impose or prescribe fixed parameters, but ask and trigger replies and solutions from the learners, which function best on the learner's own terms - however innovative or traditional."

9 "Learning to listen, learning to use tones of voice to elicit elaboration or clarification, and using pausing techniques as a reflecting tool are crucial to a good advising session."

${ }^{10}$ Centre de Recherches et d'Applications Pédagogiques en Langues, équipe du laboratoire ATILF/CNRS de l'Université Nancy 2
} 
consulter un historique des supports utilisés, gérer son rythme et ses activités via un agenda personnel, ou encore rechercher librement des matériels dans une médiathèque aux critères de recherche variés. La structure élaborée pour le site Internet tente de conceptualiser les principes pédagogiques à l'origine de la structure du dispositif afin de renforcer la conceptualisation de l'apprenant. Systématiser les modalités de l'apprentissage constitutives du contrôle pédagogique à travers les actions à entreprendre dans le site Internet apparait comme une intention forte des concepteurs. En termes d'accompagnement, les ressources humaines disponibles à l'apprenant sont d'une part, du personnel commercial avec qui l'apprenant décidera des termes de son contrat, du personnel d'accueil sensé l'orienter durant ses séances au centre, des conseillers méthodologiques ayant pour fonction de l'aider dans son projet d'apprentissage et des locuteurs natifs avec qui pratiquer la langue cible. Ces personnels, conseillers et anglophones, pourront être consultés en rendez-vous individuels ou animeront des ateliers de groupe.

Pour aborder le dispositif conçu et ses intentions d'usage, nous avons conçu une grille d'entretien semi-dirigé susceptible de faire décrire par les concepteurs les différentes composantes du dispositif, en nous fondant sur des paramètres établis (Glikman, 2002, 78 ; Jézégou, 2004 ; Denis, 2003). Les entretiens ont été réalisés auprès de deux enseignantschercheurs encadrant la conception du projet, et de deux acteurs clés de la conception générale du dispositif : le mandataire lui-même chargé du développement financier et de la coordination générale des équipes pédagogiques et techniques, et la future directrice de centre chargée du développement commercial du produit et des recrutements et formations des futurs vendeurs. Une analyse de discours a ensuite été effectuée, afin de relever thématiquement les propos des uns et des autres. Cette étape du travail a permis de faire émerger des points de consensus autour des composantes du dispositif et de la scénarisation pédagogique, ainsi que des priorités selon les différents concepteurs. Sur le plan de la vision et de la visée du dispositif (Bernard, 1999), des valeurs et des principes attachés au dispositif se dégagent avec deux centrations différentes observables : l'une sur la qualité, le professionnalisme, l'originalité, la nouveauté ; l'autre sur l'autonomisation, le respect, les relations symétriques dans les échanges et la perspective d'une formation à la formation. L'enjeu de la conception de ce dispositif est donc de répondre aux besoins d'un acteur à la fois client et apprenant, et de faire coïncider une démarche de formation (à l'anglais et à la formation) et une démarche de service, voire d'industrialisation de la connaissance.

Pour analyser les usages qui seront faits de ce dispositif, nous avons choisi de nous appuyer sur la notion d'appropriation d'un dispositif d'apprentissage, telle qu'elle a été développée par Paquelin $(2004,2009)$, et de chercher à appréhender différents « états » du dispositif, selon son hypothèse, à savoir les états prescrit, perçu, prévu et vécu. A ce stade de la recherche, nous tenterons de fonder notre appréhension du dispositif prescrit, c'est-àdire " le dispositif tel qu'il est conçu par les concepteurs et porteur d'une prescription ou intention d'usage » (Paquelin, 2004, 161) et tenterons de concevoir des hypothèses sur les dispositifs perçus et vécus potentiellement par les acteurs du dispositif, à savoir apprenants/clients et personnels pédagogiques et commerciaux.

Nous avons ainsi reproduit le protocole construit par Jézégou (2004) pour tenter d'évaluer l'ouverture du dispositif à partir de la vision de ces mêmes concepteurs, et délimiter une zone susceptible de circonscrire la prescription potentielle du dispositif. Ce protocole 
nécessite une phase de concertation entre concepteurs pour valider les termes de la grille et déterminer les pourcentages d'activités pédagogiques concernés par chacun des critères. $A$ l'issue de cette étape, un questionnaire a été co-créé avec l'équipe de concepteurs, en des termes adaptés au langage de ces acteurs et au dispositif, puis ce questionnaire a été évalué individuellement par chacun des concepteurs ${ }^{11}$. Ce travail a permis de caractériser le dispositif comme ouvert à 78,16 \%, (fig. 1), ce qui correspond à un dispositif hautement ouvert d'après la classification établie par Jézégou ${ }^{12}$. La ligne fixée par la moyenne de l'évaluation des concepteurs délimite le potentiel de libertés de choix offert à l'apprenant pour chacune des composantes du dispositif mais aussi l'extrémité supérieure d'une Zone Proximale d'Actualisation potentielle du dispositif, selon l'hypothèse de Paquelin $(2004,172)$, un « espace qui détermine l'ouverture prescrite, autorisée par les concepteurs et prescripteurs ».

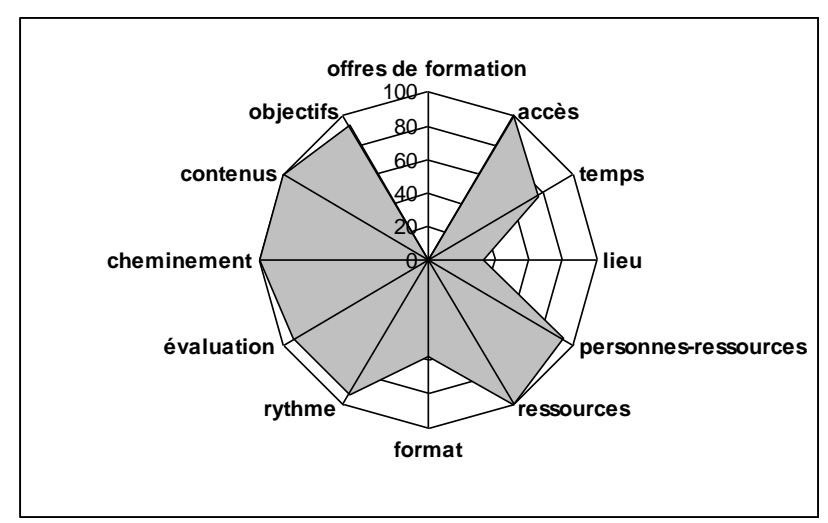

Figure 1 : Evaluation de l'amplitude des libertés de choix offertes aux apprenants du dispositif par les concepteurs (en \%)

La mise en parallèle de ces résultats aux analyses de discours portant sur les entretiens réalisés permet d'expliciter les composantes du dispositif dans leur état " prescrit ». Au sein des composantes socio-organisationnelles, les concepteurs ont ainsi reconnu que l'accès serait totalement libre, le dispositif n'imposant aucun pré-requis concernant les compétences acquises au préalable en langue anglaise. La prise de contrôle potentielle de l'apprenant sur les décisions d'orientation méthodologique prises par l'institution est en revanche évaluée comme nulle, l'apprentissage auto-dirigé avec soutien étant la seule proposition de formation faite à l'apprenant. Aucun enseignement ne sera proposé.

Les composantes spatio-temporelles seront conditionnées par certains aspects institutionnels. Un contrat sera en effet établi avec l'apprenant et définira la durée de son apprentissage, et ses activités pédagogiques dans le centre seront restreintes par l'amplitude des horaires d'ouverture, la disponibilité des différents intervenants pour des rendez-vous, et les plannings établis pour des ateliers. Des limites existeront donc nécessairement au regard des ces différents éléments, mais de larges possibilités de choix seront néanmoins offertes quant à la gestion des temps et durées de l'apprentissage, ce qui

\footnotetext{
${ }^{11}$ Pour le détail du protocole, voir Jézégou, 2005 p.122-136

12 Nous avons cependant dû apporter une modification au protocole : la composante «méthodes » n'a pas été comprise par les concepteurs et a dû être exclue au cours de la conception de la grille d'analyse.
} 
a amené les concepteurs à évaluer cette composante comme ouverte à $75 \%$. Les lieux d'apprentissage n'ont été évalués comme ouverts qu'à $33 \%$, car ils seront conditionnés par les orientations prises par les concepteurs : le centre de ressources est vu comme un lieu de vie et d'échanges potentiellement vecteur de motivation et d'expériences sociales, et sa fréquentation est donc fortement souhaitée, malgré la possibilité de recourir à de nombreuses ressources d'apprentissage via une plate-forme numérique en ligne. Ainsi, les activités sociales, rendez-vous et ateliers auront nécessairement lieu au centre, et le travail de l'apprenant sur les ressources est fortement souhaité dans les locaux.

Les ressources pédagogiques offertes aux apprenants sont agencées au sein du site Internet selon le respect de critères de centres de ressources. Pour prendre en compte la diversité des utilisateurs et l'hétérogénéité du public, et permettre à chacun " d'en faire l'usage qu'il souhaite en faire », les matériels doivent satisfaire trois conditions principales : « être adaptables, autosuffisants et accessibles "(Carette et Holec, 1995). L'adaptabilité repose sur la " non-préadaptation ", c'est-à-dire sur la possibilité d'exploiter les ressources pédagogiques selon les objectifs choisis par l'apprenant, et non des objectifs imposés par les concepteurs d'activités. Ce critère a été rempli dans une certaine mesure, les ressources du centre étant composées de matériels didactiques du commerce, pré-adaptés, de documents authentiques accompagnés ou non d'activités autocorrectives, de conseils d'exploitation, afin d'offrir un continuum possible du « faire apprendre » au «laisser apprendre » (Puren, 2004, 244). A cela s'ajoute la possibilité d'intégrer à l'apprentissage des ressources personnelles aux apprenants. L'autosuffisance des documents, en particulier des documents authentiques, repose sur l'accès à des aides et des références disponibles. Pour cela, les transcriptions des documents audio ou vidéo sont toujours disponibles, ainsi que différentes aides (dictionnaires, encyclopédies etc.). L'accessibilité tient à l'agencement d'une base de données pourvue de critères de description nombreux et pertinents, et à la possibilité d'une recherche multicritères au sein d'une interface formatrice et transparente (Cembalo, 1995, 96). L'aspect formateur de l'accessibilité des documents a lui aussi été conçu selon le continuum " faire apprendre " à " laisser apprendre " en permettant l'accès à des ressources suggérées automatiquement selon les objectifs des apprenants, et à une médiathèque aux critères de recherches manipulables librement. Avec près d'un millier de documents authentiques majoritairement sonores ou vidéo, et un demi-millier d'activités pédagogiques issues de CD-ROM ou conçues pour l'exploitation des documents authentiques du site Internet, les concepteurs ont jugé la liberté d'accès aux ressources ainsi définie comme totale.

Les apprenants pourront recourir au soutien de différentes personnes-ressources. Un conseiller sensé jouer les rôles et posséder les compétences didactiques et interactionnelles évoqués précédemment pourra intervenir au cours d'entretiens de conseil individuels ou d'ateliers méthodologiques de groupe. Pour la pratique linguistique, des entretiens individuels avec des anglophones seront proposés ainsi que des ateliers thématiques de groupe. Au cours du travail individuel sur les ressources, le personnel d'accueil du centre pourra être sollicité pour résoudre des problèmes. Cette configuration prévue a conduit les concepteurs à évaluer l'accès aux personnes ressources comme ouvert à plus de $91 \%$. Les analyses de discours font cependant émerger des réserves : l'apprenant connaîtra différentes limites dans la sollicitation des personnes-ressources, en terme de durée et de fréquence des entretiens. 
Le format - travail seul, en binôme, en groupe - est potentiellement varié grâce à la possibilité de choix et de combinaisons entre entretiens individuels ou ateliers de groupe. L'évaluation de cette composante est cependant limitée à une ouverture à $58 \%$, le travail individuel sur les matériels et le site Internet étant prévu comme une part importante de la formation.

Les derniers critères, à savoir la définition des objectifs, des contenus, du cheminement, du rythme et de l'évaluation de l'apprentissage dépendront des décisions de l'apprenant, assisté du conseiller pédagogique et éventuellement d'anglophones dans la co-construction de son projet. Si l'amplitude est presque maximale pour chacun de ces critères, c'est bien parce que la prise de contrôle de l'apprenant sur l'apprentissage est fortement souhaitée, par les concepteurs pédagogiques qui visent l'autonomisation de l'apprenant dans le système, et par les concepteurs attachés à la commercialisation du dispositif, qui voient une plus-value intéressante à une individualisation réelle des parcours.

\section{Conclusion}

Un des paramètres susceptibles de rendre compte de la distance transactionnelle inhérente à l'état prévu de ce dispositif a donc été évalué, à travers son degré général d'ouverture et l'évaluation de la liberté de choix accordée à l'apprenant dans la gestion de chacune de ses composantes. Ces libertés peuvent être entravées par des impératifs économiques ou par des décisions prises en termes de choix méthodologiques ou logistiques par les concepteurs. L'analyse réalisée de l'état prescrit du dispositif nous permet ainsi d'appréhender la variable de l'ouverture avec une relative précision. En revanche, si des hypothèses ont été faites sur la variable dialogue au sein de ce dispositif, à travers les caractéristiques possibles et souhaitables d'entretiens de conseil visant l'autonomisation de l'apprenant, respectant sa prise de décision, étayant son champs des possibles, et participant à une actualisation optimale des différentes composantes du dispositif, les états perçus et vécus pourront seuls nous permettre de réellement évaluer d'une part la qualité du dialogue entrepris, et d'autre part l'autonomisation des apprenants.

Ce dispositif s'inscrit dans un continuum entre qualité de service et éthique pédagogique, et il est probable que la représentation du dispositif « à prescrire » diffère entre la vision des différents concepteurs et la prescription opérée par les personnels commerciaux et pédagogiques. L'état " perçu » du dispositif par ces personnels pourrait influencer l'état « perçu » des apprenants d'une manière encore imprévisible aujourd'hui. D'autre part, les dialogues pédagogiques qui soutiendront les projets de formation des apprenants pourront ou non concourir à une réduction significative de la distance transactionnelle, s'ils répondent aux critères de qualité et de présence déterminés. Mais entre qualité pédagogique et qualité de service, c'est-à-dire respect des objectifs et des cahiers des charges de l'entreprise, des tensions peuvent apparaître, et la mise en œuvre de l'accompagnement des conseillers pourrait différer en profondeur des attentes des concepteurs. Des travaux ultérieurs seront donc entrepris pour analyser d'une part l'appropriation du dispositif par les apprenants, à travers leur utilisation effective du site Internet et des différents éléments du dispositif, mais aussi à travers leur représentation de celui-ci à différents stades de leur formation. Nous espérons que l'analyse d'interactions de conseil nous permettra enfin de mieux étudier les rapports entre ouverture, dialogue et prise de contrôle par l'apprenant de son projet de 
formation, afin de continuer à mieux explorer l'hypothèse qu'un dispositif à la structure ouverte et au dialogue fréquent et de qualité peut concourir à l'autonomisation des apprenants, et favoriser les processus de distanciation et d'acquisition de compétences d'apprentissage. 


\section{Bibliographie}

Bernard M., Penser la mise à distance en formation, Paris, L'Harmattan, 1999.

Bouchard P., " Autonomie et distance transactionnelle dans la formation à distance ", in Cyberespace et formations ouvertes : vers une mutation des pratiques de formation ?, Perspectives en éducation et formation, Bruxelles, De Boeck Université, 2000, p. 65-78.

Carette E. \& Castillo D., "Devenir conseiller : quels changements pour l'enseignants ? », Mélanges CRAPEL, $\mathrm{n}^{\circ}$ 27, 2004, p. 71-97.

Carette E. \& et Holec H., "Quels matériels pour les centres de ressources ? », Mélanges CRAPEL, $\mathrm{n}^{\circ} 22,1995$, p. 85-94.

Cembalo S.-M., « Le catalogage, l'indexation et les fichiers dans les centres de ressources en langues ", Mélanges CRAPEL, n²2, 1995, p. 95-104.

Ciekanski M., L'accompagnement à l'autoformation en langue étrangère : contribution à l'analyse des pratiques professionnelles : Etude des dimensions langagières et formatives des pratiques dites "de conseil" dans des systèmes d'apprentissage autodirigé en langue étrangère, Thèse de doctorat, Université Nancy 2, 2005.

Ciekanski M., " Fostering learner autonomy: power and reciprocity in the relationship between language learner and language learning adviser ", Cambridge Journal of Education, vol. 37, $\mathrm{n}^{\circ} 1,2007$, p. 111-127.

Denis B., "Quels rôles et quelle formation pour les tuteurs intervenant dans des dispositifs de formation à distance ? », Distances et savoirs, vol. 1, n 1, 2003, p. 19-46.

Garrison D. \& Anderson T., E-learning in the 21st century : a framework for research and practice, London, Routledge Falmer, 2003.

Glikman V., Des cours par correspondance au e-learning : panorama des formations ouvertes et à distance, Paris, Presses universitaires de France, 2002.

Gorsky P. et Caspi A., 2005, " A critical analysis of transactional distance theory », The Quarterly Review of Distance Education, vol. 6, $\mathrm{n}^{\circ}$ 1, p. 1-11.

Gremmo M-J., « Conseiller n'est pas enseigner : Le rôle du conseiller dans l'entretien de conseil. ", Mélanges CRAPEL, n²2, 1995, p. 33-61.

Holec H. \& Cembalo S-M., "Les langues aux adultes : pour une pédagogie de l'autonomie », Mélanges CRAPEL, 1973.

Holec $\mathrm{H}$., Autonomie et apprentissage des langues étrangères, Conseil de la coopération culturelle, Strasbourg, 1979.

Jacquinot G., " Apprivoiser la distance et supprimer l'absence ? Ou les défis de la formation à distance ", Revue française de pédagogie, n 102, 1993, p. 55-67.

Jézégou A., « La distance en formation. Premier jalon pour une opérationnalisation de la théorie de la distance transactionnelle ", Distances et savoirs, vol. 5, n 3, 2007, p. 341366. 
Jézégou A., "Apprentissage autodirigé et formation à distance », Distances et savoirs, vol. 6, n 3, 2008, p. 343-364.

Jézégou A., Formations ouvertes : libertés de choix et autodirection de l'apprenant, Paris, l'Harmattan, 2005.

Jézégou A., Formation ouverte et stratégies d'autodirection, Thèse de doctorat, Université de Paris-Nanterre, 2004.

Jézégou A., «Formations ouvertes et autodirection : pour une articulation entre libertés de choix et engagement cognitif de l'apprenant ", Education permanente, $n^{\circ} 152,2002, \mathrm{p}$. 43-53.

Linard M., " Autoformation, éthique et technologies : enjeux et paradoxes de l'autonomie ", in Autoformation et enseignement supérieur, Paris, Hermès-Lavoisier, 2003, p. 241-263.

Linard M., «La distance en formation : une occasion de repenser l'acte d'apprendre », Accès à la Formation à Distance, Clés pour un Développement Durable, Geneva 10-12, Octobre 1994, p. 46-55.

Moore M-G., «Theory of transactional distance », in Theoretical principles of distance education, Routledge, 1993, p. 22-38.

Moore M-G., "Towards a theory of independent learning and teaching. », Journal of Higher Education, $\mathrm{n}^{\circ} 44,1973$, p. 661-679.

Mozzon-McPherson M., «Supporting Independent Learning Environments: An Analysis of Structures and Roles of Language Learning Advisers ", System: An International Journal of Educational Technology and Applied Linguistics, vol. 35, n 1, 2007, p. 66-92.

Paquelin D., L'appropriation des dispositifs numériques de formation : du prescrit aux usages, Paris, l'Harmattan, 2009.

Paquelin D., «Le tutorat : accompagnement de l'actualisation du dispositif », Distances et savoirs, vol. 2, n²-3, 2004, p. 157-182.

Poteaux N., " Du dispositif ouvert au dispositif à distance : étude de cas pour une nouvelle transposition ", in A distance : apprendre, travailler, communiquer, Strasbourg, Presses universitaires de Strasbourg, 2007, p. 77-89.

Puren C., "Quels modèles didactiques pour la conception de dispositifs d'enseignement / apprentissage en environnement numérique ? », ELA, vol. 2, n 134, 2004, p. 235-249.

Rabardel P., Les hommes et les technologies : approche cognitive des instruments contemporains, Paris, A. Colin, 1995. 